\title{
PROGRAMA PARA ESTIMATIVA DA UMIDADE DE EQUILÍBRIO DA MADEIRA
}

\section{A PROGRAM TO ESTIMATE THE EQUILIBRIUM MOISTURE CONTENT OF WOOD}

\author{
Jackson Roberto Eleotério $^{1}$ Clóvis Roberto Haselein ${ }^{2}$ Nestor Pedro Giacomini ${ }^{3}$
}

\begin{abstract}
RESUMO
Neste trabalho, um programa para calcular a umidade de equilíbrio da madeira é introduzido. O programa, em linguagem Delphi, foi desenvolvido em dois módulos, utilizando como base as equações de Hailwood-Horrobin de um e dois hidratos.
\end{abstract}

Palavras-chave: Umidade de equilíbrio da madeira, secagem.

\begin{abstract}
In this article, a program to calculate the wood equilibrium moisture content is introduced. The program was developed in two modulii, in Delphi language, using the one and two-hidrate forms of the Hailwood-Horrobin equations.

Key words: Wood equilibrium moisture content, drying.

\section{INTRODUÇÃO}

A madeira exposta ao ar perde ou ganha umidade até atingir um equilíbrio dinâmico, conhecido como umidade de equilíbrio. Por esta razão, a umidade de equilíbrio é um fator importante que deve ser considerado na utilização da madeira exposta ao ar. O assentamento de qualquer peça de madeira, cujo teor de umidade não esteja em equilíbrio com as condições reinantes no local de aplicação poderá resultar em defeitos tais como frestas em assoalhos, empenos de paredes e portas, ou, mesmo, em esforços (tensões) em elementos estruturais.

$\mathrm{Na}$ secagem da madeira, a umidade de equilíbrio é uma das bases na elaboração de

1. Eng. Florestal, Pós-Graduando pela ESALQ/USP. CEP: 13418-110. Piracicaba. SP.

2. Eng. Florestal, PhD., Prof. Adjunto do Departamento de Ciências Florestais/CCR/UFSM. CEP: 97105-900. Santa Maria. RS.

3. Eng. Químico. CT/UFSM. CEP: 97105-900. Santa Maria. RS.
\end{abstract}


programas; por exemplo programas baseados no potencial de secagem. Em geral, quanto maior a relação entre umidade atual e umidade de equilíbrio da madeira, mais rápido o processo de secagem.

Apesar da heterogeneidade da madeira quanto à espécie, extrativos, história da exposição, etc., a umidade de equilíbrio da madeira exposta ao ar pode ser prevista hoje com bastante precisão para a maioria das condições (DURLO, 1991). Exemplo disso são as tabelas de umidade de equilíbrio disponíveis para muitas cidades do país (LIMA et al., 1995; SANTINI, 1984). Tabelas estão disponíveis também para madeira submetida à secagem artificial, cuja temperatura é, em geral, superior à ambiental (GALVÃO \& JANKOWSKY, 1985; CECH \& PFAFF, 1977; RASMUSSEN, 1961). Mesmo para madeira sendo seca a altas temperaturas (acima de $100^{\circ} \mathrm{C}$ ) existem tabelas com precisão adequada.

Normalmente, a umidade de equilíbrio da madeira é fornecida em função da temperatura e da umidade relativa do ambiente a que a madeira está exposta. No processo de secagem, o mais comum é encontrar-se estas tabelas fornecidas em função da temperatura dos termômetros de bulbo seco e de bulbo úmido, dada a sua relação com a umidade relativa.

O objetivo do presente trabalho é discorrer brevemente sobre as equações existentes para predizer a umidade de equilíbrio da madeira, a umidade relativa, as pressões parciais de vapor e de saturação ao ar. Hoje estas são mais adequadas do que a consulta a tabelas. No final, um pequeno algorítimo será fornecido como exemplo e que poderá ser modificado para atender finalidades específicas do usuário.

\section{UMIDADE RELATIVA}

A umidade relativa pode ser expressa pela relação percentual entre a pressão parcial e a pressão de saturação de vapor de água à mesma temperatura:

$$
h=\frac{p}{p_{s}}
$$

Onde: $\mathrm{h}=$ pressão relativa;

$\mathrm{p}=$ pressão parcial de vapor;

$\mathrm{p}_{\mathrm{s}}=$ pressão de saturação de vapor;

e a umidade relativa $\mathrm{UR}=\mathrm{h} \times 100$.

Tabelas para UR utilizadas na secagem da madeira comumente são encontradas em função da diferença entre as temperaturas de bulbo seco e bulbo úmido. Uma equação empírica para pressão parcial de vapor pode ser da forma (MÜLLER, 1989):

$$
p=4.5825 \times 10^{\left(\frac{7.5 T_{b u}}{237,5+T_{b u}}\right)}-0,6\left(T_{b s}-T_{b u}\right)
$$




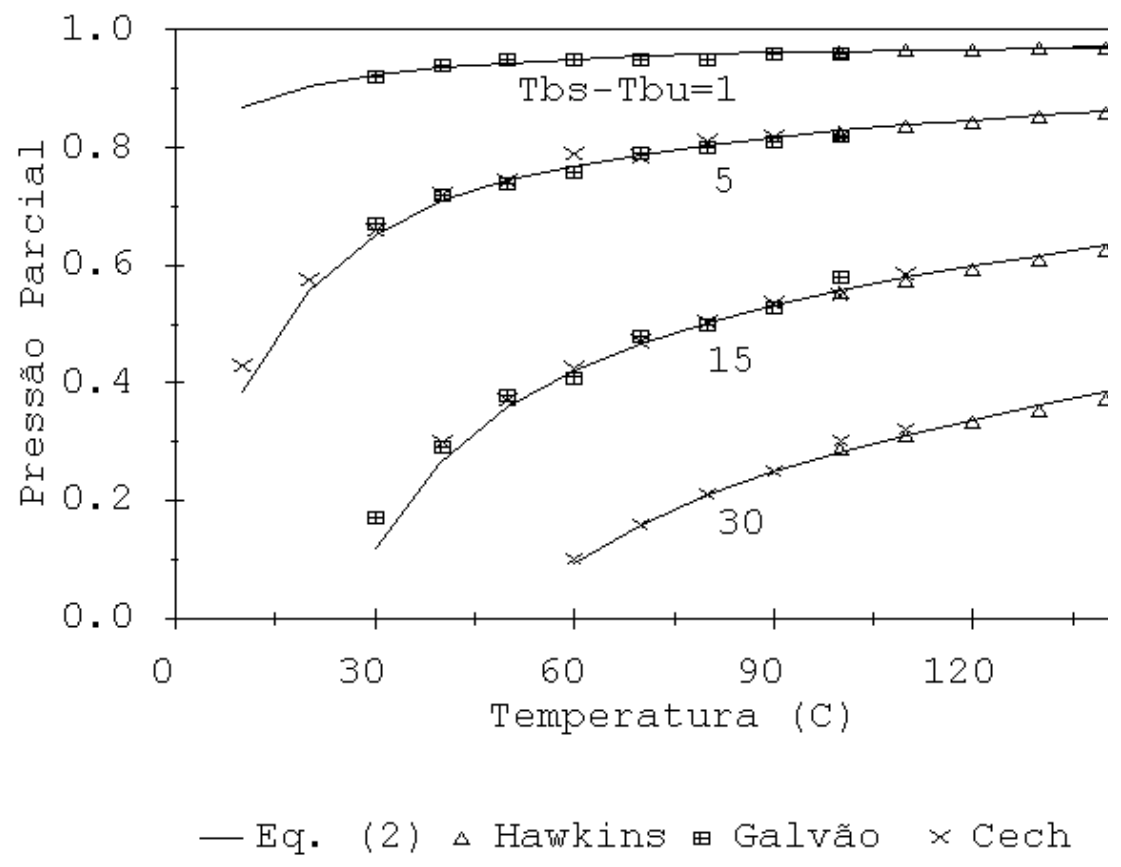

FIGURA 1: Pressão parcial de vapor em função da temperatura de bulbo seco para algumas diferenças higrométricas.

Para temperaturas acima de $100^{\circ} \mathrm{C}$, SIMPSON \& ROSEN (1981) adotam o procedimento de HAWKINS (1978), através de um balanço de massa e energia para o processo de saturação adiabática:

$$
Y=Y^{*}-\frac{\left(0,24+0,44 Y_{s}\right)\left(T_{b s}-T^{*}\right)}{1094+0,44 T_{b s}-T^{*}}
$$

Onde:

Y = umidade específica (libras água/libras de ar seco);

$\mathrm{Y}^{*} \quad=$ umidade específica para saturação a $\mathrm{T}_{\mathrm{s}}$ (libras água/libras de ar seco);

$\mathrm{T}_{\mathrm{bs}} \quad=$ temperatura de bulbo seco $\left({ }^{0} \mathrm{~F}\right)$;

$\mathrm{T}^{*} \quad=$ temperatura de saturação adiabática $\left({ }^{0} \mathrm{~F}\right)$;

Ys = umidade específica para saturação a temperatura de saturação adiabática (libras de água / libras de ar seco);

Sendo: $Y_{S}=\frac{p^{*}}{1,61\left(p_{T}-p^{*}\right)}$

$\mathrm{p}^{*}=$ pressão de vapor a $\mathrm{T}^{*}$ (polegadas de mercúrio);

$\mathrm{p}_{\mathrm{T}}=$ pressão total (polegadas de mercúrio) 
Para calcular a pressão parcial de vapor a $\mathrm{T}_{\mathrm{bs}}$ e $\mathrm{T}^{*}$ é necessário calcular a pressão parcial de vapor $\mathrm{p}$ a $\mathrm{T}_{\mathrm{bs}}$ e $\mathrm{T}^{*}$ :

$$
p=\frac{1,61 Y p_{T}}{1+1,61 Y}
$$

e a pressão relativa de vapor

$$
h=\frac{p}{p_{s}}
$$

Onde: $p_{s}=$ pressão de saturação a $\mathrm{T}_{\mathrm{bs}}$ (polegadas de mercúrio).

A umidade relativa é então definida por $U R=h \times 100$, da mesma forma como na equação 1 . Este procedimento é baseado na discussão de ROSEN \& SIMPSON (1980) que consideram que na faixa de 215 a $300^{\circ} \mathrm{F}$ as diferenças entre a verdadeira temperatura de bulbo úmido e a temperatura de bulbo úmido termodinâmica são negligenciáveis. A Figura 1 mostra graficamente a Equação 2 para $1,5,15$ e $30^{\circ} \mathrm{C}$ de diferença entre TBS e TBU juntamente com pressões parcias provenientes de várias outras fontes (HAWKINS, 1978; GALVÃO \& JANKOWSKY, 1985; CECH \& PFAFF, 1977).

Já a pressão de saturação de vapor $\left(\mathrm{p}_{\mathrm{s}}\right)$ pode ser obtida a partir da temperatura usando-se uma equação do tipo Kirchof (SKAAR, 1988; ASHRAE HANDBOOK, 1985):

$$
\operatorname{Ln}\left(\mathrm{p}_{\mathrm{s}}\right)=\mathrm{c}_{0}+\mathrm{c}_{1} \mathrm{~T}+\mathrm{c}_{2} \mathrm{~T}^{2}+\mathrm{c}_{3} \mathrm{~T}^{3}+\frac{\mathrm{C}_{4}}{\mathrm{~T}}+\mathrm{c}_{5} \operatorname{Ln}(\mathrm{T})
$$

Onde: $\quad c_{o}=1,3914993$;

$$
\begin{aligned}
& \mathrm{c}_{1}=-0,048640239 ; \\
& \mathrm{c}_{2}=4,1764768 \times 10^{-5} ; \\
& \mathrm{c}_{3}=-1,4452093 \times 10^{-8} ; \\
& \mathrm{c}_{4}=-5800,2206 ; \\
& \mathrm{c}_{5}=6,5459673 ; \\
& \mathrm{T}=\text { temperatura absoluta }\left({ }^{0} \mathrm{~K}\right) .
\end{aligned}
$$

A pressão total pode ser aproximada usando-se a lei de Dalton de pressões parciais e a Lei Geral dos gases ideais (uma aproximação satisfatória para a maioria das aplicações).

$$
\begin{aligned}
p_{\mathrm{H}_{2} \mathrm{O}} & =\rho_{\mathrm{H}_{2} \mathrm{O}} R_{\mathrm{H}_{2} \mathrm{O}} T \\
p_{\mathrm{ar}} & =\rho_{\mathrm{ar}} \mathrm{R}_{\mathrm{ar}} T
\end{aligned}
$$

Onde: $\quad \mathrm{P}_{\mathrm{H} 2 \mathrm{O}} \quad=$ pressão parcial de vapor $(\mathrm{Pa})$;

$\mathrm{p}_{\mathrm{ar}} \quad=$ pressão parcial do ar seco $(\mathrm{Pa})$;

$\rho_{\mathrm{H} 2 \mathrm{O}}=$ densidade vapor $\left(\mathrm{kg} / \mathrm{m}^{3}\right)$; 


$$
\begin{array}{ll}
\rho_{\mathrm{ar}} & =\text { densidade } \operatorname{ar}\left(\mathrm{kg} / \mathrm{m}^{3}\right) ; \\
\mathrm{R}_{\mathrm{H} 2 \mathrm{O}} & \sim 462 \mathrm{~J} / \mathrm{kg}^{0} \mathrm{~K}(\text { constante para o vapor de água); } \\
\mathrm{R}_{\mathrm{ar}} & \sim 287 \mathrm{~J} / \mathrm{kg}^{0} \mathrm{~K}(\text { constante para o ar seco); } \\
\mathrm{T} & =\text { temperatura absoluta }\left({ }^{0} \mathrm{~K}\right) .
\end{array}
$$

Pela lei de Dalton para pressão parcial, $\mathrm{p}_{\mathrm{T}}=\mathrm{p}+\mathrm{p}_{\mathrm{ar}}$.

\section{UMIDADE DE EQUILÍBRIO PARA A MADEIRA}

SKAAR (1972) apresenta uma revisão sobre as teorias existentes para previsão da umidade de equilíbrio da madeira. A teoria BET (Brunauer, Emmet \& Teller) utiliza o conceito de camadas. Uma camada monomolecular firmemente aderida às hidroxilas da parede celular e camadas secundárias diferentes da camada monomolecular mas de comportamento termodinâmico idêntico. Esta teoria de multicamadas é uma modificação da teoria de camada única desenvolvida por Langmuir:

$$
\frac{h}{M(1-h)}=\frac{1}{M_{m} C}+\frac{(C-1) h}{M_{m} C}
$$

Onde:

$\mathrm{M}=$ teor de umidade da madeira;

$\mathrm{M}_{\mathrm{m}}=$ teor de umidade da madeira correspondente a uma sorção monomolelcular completa;

$\mathrm{C}=$ constante $(\sim 6)$.

O modelo da equação 9 inclui número infinito de camadas de moléculas de água, tendo sido modificada pelos autores para incluir um número múltiplo finito de camadas (n) de moléculas de água. Os melhores ajustes são conseguidos com n variando de 6 a 8 (SKAAR, 1972):

$$
\frac{h}{M(1-h)}=\frac{1}{M_{m} C} \frac{1+(C-1) h-C h^{n+1}}{1-(n+1) h^{n}+n h^{n+1}}
$$

O modelo desenvolvido por Hailwood e Horrobin difere da teoria de camadas Langmuir, BET e Dent por considerar que o processo de sorção é governado pela formação de uma solução sólida de água no polímero e a formação de hidratos entre água e unidades definidas da molécula do polímero hidratado. A mistura de polímero, polímero hidratado e água dissolvida formam uma única fase sólida. Os dois componentes água e polímero, existem em duas fases, e as moléculas de água dissolvidas na fase sólida são consideradas móveis. A equação geral de múltiplos hidratos desenvolvida por Hailwood e Horrobin é a seguinte (SKAAR, 1972):

$$
\mathrm{u}_{\mathrm{e}}(\%)=\frac{18}{\mathrm{~W}}\left[\frac{\mathrm{Kh}}{1-\mathrm{Kh}}+\frac{\mathrm{KhK}_{1}+2 \mathrm{~K}_{1} \mathrm{~K}_{2} \mathrm{~K}^{2} \mathrm{~h}^{2}}{1+\mathrm{KhK}_{1}+\mathrm{K}_{1} \mathrm{~K}_{2} \mathrm{~K}^{2} \mathrm{~h}^{2}}\right] \times 100
$$


Sendo que:

$$
\mathrm{u}_{\mathrm{e}}(\%)=\frac{18}{\mathrm{~W}}\left[\frac{\mathrm{Kh}}{1-\mathrm{Kh}}+\frac{\mathrm{KhK}_{1}}{1+\mathrm{KhK}_{1}}\right] \times 100
$$

é conhecida como a equação de um hidrato e a anterior de dois hidratos. O primeiro termo da equação entre parêntesis representa a fração da água livre dissolvida enquanto que os demais termos representam a fração da água presente como monohidrato, dihidrato, etc; $\mathrm{K}$ é a constante de equilíbrio entre a água dissolvida e a sua pressão relativa de vapor $(h) ; K_{\mathrm{i}}$ é constante de equilíbrio, representado pela razão entre a atividade química do hidrato para as atividades dos dois reagentes (água e madeira não-hidratada); 18 é o peso molecular da água e W o peso molecular da madeira por mol de sítio de adsorção.

SIMPSON (1973) e SIMPSON \& ROSEN (1981) determinaram os coeficientes destas equações através de regressão não-linear, utilizando os dados da tabela de umidade de equilíbrio publicada no WOOD HANDBOOK (1955) para madeira de Sitka spruce (Picea sitchensis). A equação de dois hidratos foi considerada superior na predição da umidade de equilíbrio da madeira. O WOOD HANDBOOK (1987) revisou os coeficientes desta equação e publicou uma nova tabela baseada nos dados ajustados. Apesar de ser ajustada para apenas uma espécie de madeira, com valores de umidade de equilíbrio intermediários entre os extremos de histeresis para cada umidade relativa, a equação e a tabela tem sido amplamente utilizadas na previsão da umidade de equilíbrio de outras madeiras e, mesmo, outros materiais, destas derivados. O ideal seria que esta equação fosse ajustada para cada material em particular.

Já para temperaturas acima de $100^{\circ} \mathrm{C}$ a adequação das equações é mais problemática. Devido à exiguidade dos dados, estas equações não foram ajustadas para estas temperaturas; normalmente usa-se extrapolações das equações ajustadas a baixas temperaturas. À pressão atmosférica, somente um número limitado de umidades relativas são possíveis, com temperaturas de bulbo úmido inferiores às de bulbo seco. A maior parte das determinações da $U_{\mathrm{e}}$ existentes atualmente é à pressão atmosférica e limitada, portanto, a temperaturas de bulbo úmido inferiores às de bulbo seco.

Um dos métodos existentes para prever a umidade de equilíbrio da madeira para temperaturas acima de $100^{\circ} \mathrm{C}$ foi desenvolvido por KAUMAN (1956) através da extrapolação da $U_{e}$ a baixas temperaturas. $\mathrm{O}$ gráfico permite a leitura da $\mathrm{U}_{\mathrm{e}}$ em função das temperaturas de bulbo seco e bulbo úmido. Já SIMPSON \& ROSEN (1981) discutem a extrapolação da equação de um hidrato ajustada para baixas temperaturas para uso a altas temperaturas. Os resultados são comparados com aqueles existentes na literatura à pressão atmosférica. A discrepância máxima encontrada foi de $2.1 \%$.

Por outro lado, para pressões acima de uma atmosfera, as previsões são incertas e, mesmo, as medições feitas até o momento são contraditórias e pouco confiáveis. Apesar da concordância de que a madeira atingirá uma umidade de equilíbrio em função da temperatura e pressões parciais de vapor, os resultados das extrapolações através dos ábacos de Kauman e das equações de um e de dois hidratos geralmente não são coincidentes, principalmente para altas umidades relativas (RESCH, 1988). Com os coeficientes apresentados no WOOD HANDBOOK (1987) a equação de dois hidratos produz valores de $\mathrm{U}_{\mathrm{e}}$ adequados para umidade de equilíbrio a temperaturas abaixo de 
$120^{\circ} \mathrm{C}$. Com os coeficientes originais determinados por SIMPSON (1973) estes são apresentados na Figura 2, para as isotermas de 120,140 e $160^{\circ} \mathrm{C}$. Aparentemente, a equação de um hidrato ajusta-se melhor aos dados de umidade de equilíbrio publicados por KAUMAN (1956) e RESCH (1988).
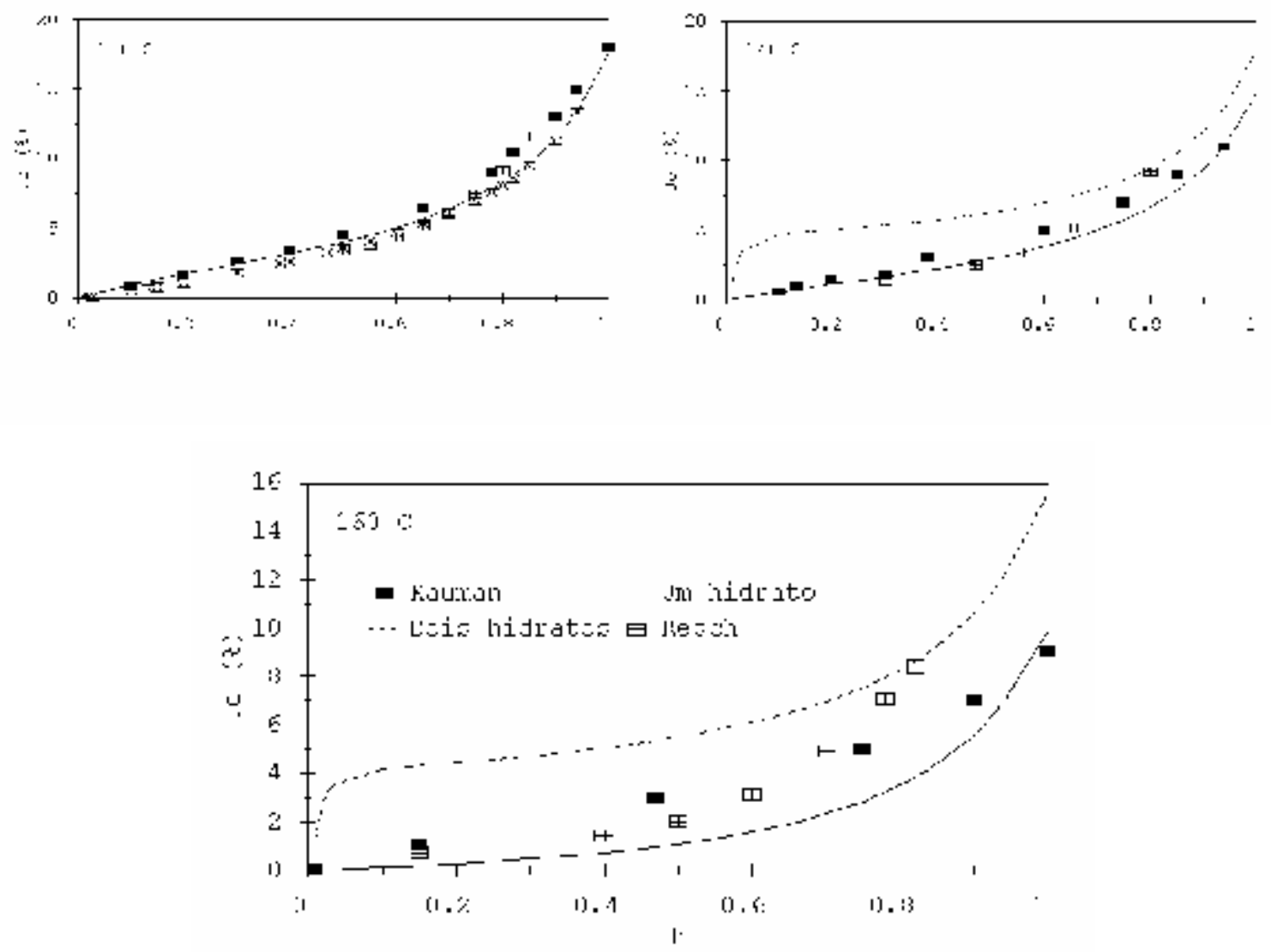

FIGURA 2: Isotermas de sorção em vapor superaquecido a temperaturas de 120,140 e $160^{\circ} \mathrm{C}\left(\mathrm{U}_{\mathrm{e}}=\right.$ Umidade de equilíbrio; $h$ = pressão relativa).

\section{PROGRAMA}

Um pequeno programa foi desenvolvido baseado nas seguintes considerações:

a) abaixo de $100^{\circ} \mathrm{C}$ a equação de dois hidratos é usada para estimar a $\mathrm{U}_{\mathrm{e}}$. Os coeficientes são aqueles publicados no WOOD HANDBOOK (1987); 


$$
\begin{aligned}
& \mathrm{W}=330+0,452 \mathrm{~T}+0,00415 \mathrm{~T}^{2} \\
& \mathrm{~K}=0,791+0,000463 \mathrm{~T}-0,000000844 \mathrm{~T}^{2} \\
& \mathrm{~K}_{1}=6,34+0,000775 \mathrm{~T}-0,0000935 \mathrm{~T}^{2} \\
& \mathrm{~K}_{2}=1,09+0,0284 \mathrm{~T}-0,0000904 \mathrm{~T}^{2}
\end{aligned}
$$

b) acima de $100^{\circ} \mathrm{C}$ a equação de um hidrato é usada. Os coeficientes são aqueles determinados por Simpson, 1971:

$$
\begin{aligned}
& \mathrm{W}=216,9+0,01961 \mathrm{~T}+0,00572 \mathrm{~T}^{2} \\
& \mathrm{~K}=0,674+0,001053 \mathrm{~T}-0,000001714 \mathrm{~T}^{2} \\
& \mathrm{~K}_{1}=3,73+0,03642 \mathrm{~T}-0,0001547 \mathrm{~T}^{2}
\end{aligned}
$$

Onde: $\mathrm{T}=$ temperatura $\left({ }^{0} \mathrm{~F}\right)$;

c) a pressão parcial de vapor é dada pela equação 2 ;

d) a pressão de saturação de vapor é calculada pela equação 7 .

O programa foi desenvolvido em linguagem Delphi, a partir de uma versão para DOS, desenvolvida em linguagem Pascal. O programa está dividido em dois módulos, partindo de uma tela de apresentação, onde estes podem ser selecionados.

No MÓDULO I pode-se determinar, em função das temperaturas de bulbo seco e de bulbo úmido em graus Celsius, a UE e a umidade relativa do ambiente nas condições estabelecidas.

Com o MÓDULO II substitui-se o uso de tabelas e de interpolações para a determinação da temperatura de bulbo úmido, quando são dadas a temperatura de bulbo seco em ${ }^{\circ} \mathrm{C}$ e a umidade de equilíbrio em \%. Este módulo opera com as mesmas equações do módulo 1, utilizando um processo interativo de aproximação sucessiva de TBU até ser obtida uma UE com diferença máxima de 0,05\% daquela fornecida pelo operador.

O programa especifica também se as condições fornecidas pelo usuário são possíveis à pressão atmosférica. Pressões de vapor são comparadas com aquelas de uma equação do tipo de Kirchoff (SKAAR, 1972; ASHRAE HANDBOOK, 1985). Se a umidade relativa originada da combinação de TBS fornecido e TBU calculado não for possível à pressão atmosférica, é indicada a pressão em que esta situação é possível, desde que a umidade relativa não seja maior que $100 \%$. Porém, o usuário é advertido de que esta situação não é possível à pressão atmosférica.

Foi incluída uma pequena ajuda ao programa, acessada a qualquer tempo dentro do programa.

\section{CONCLUSÕES}

Criou-se um meio de facilitar o cálculo das conhecidas equações para estimativa da umidade de equilíbrio, bem como para calcular a temperatura de bulbo úmido quando são fornecidas a 
temperatura de bulbo seco e umidade de equilíbrio, facilitando e incentivando seu uso.

\section{REFERÊNCIAS BIBLIOGRÁFICAS}

ASHRAE - American Society of Heating, Refrigerating and Air-Conditioning Engineers ASHRAE Handbook of Fundamentals. Chapter 6: Psychrometrics, 1985.

CECH, M. Y.; PFAFF, F. Kiln operator's manual for eastern Canada. Report OPx192E. Ottawa, Ontario, 1977. p165-169.

DURLO, M. A. Relação água-madeira. In.: I Seminário sobre Secagem de Madeira. Santa Maria: UFSM, CEPEF/FATEC, 1991.

GALVÃO, A. P. M. de \& JANKOWSKY, I. P. Secagem racional da madeira. São Paulo: Nobel, 1985.

HAWKINS, G.A. Thermal proprerties of substances and thermodynamics. In. Mark's Standard Handbook for Mechanical Engineers. New York: Mc Graw-Hill, 8 a. ed., p. 4-31, 1978.

KAUMAN, W. G. Equilibrium moisture content relations and drying control in superheated steam drying. Forest Prod. J. 16(4):25-32, 1956.

LIMA, J.T.; MENDES, L.M.; CALEGÁRIO, N. Estimativa da umidade de equilíbrio para madeiras no Brasil. Revista da Madeira, N 23, Vol. IV. Jul/Ago 1995.

MÜLLER, P. B. Bioclimatologia aplicada aos anumais domésticos. Porto Alegre: Sulina, $3^{\mathrm{a}}$. ed., 1989.

RASMUSSEN, E.F. Dry kiln operator's manual. U.S. Dept. of Agric. Handb. 188. Washingon DC. 1961.

RESCH, H.; HOAG, M.L.; ROSEN, H.N. Desorption of yellow-poplar in superheated steam. Forest Prod. J. 38(3):13-18. 1988.

ROSEN, H. N.; SIMPSON. W. T. Evaluating Humidity at Dry-Bulb Temperatures above the Normal Boiling Point of Water. Wood Fiber, 13(1):97-101. 1980.

SANTINI, E. J. Umidade de equilíbrio para algumas cidades do Rio Grande do Sul. In.: CONGRESSO FlORESTAL ESTADUAL, 5. Anais..., v. III, p. 828-840, Nova Prata(RS), 1984.

SIMPSON, W.T. Equilibrium moisture content prediction for wood. Forest Prod. J., 21(5):48-49. 1971.

SIMPSON, W.T. Predicting equilibrium moisture content of wood by mathematical models. Wood and Fiber, 5(1):41-45. 1973.

SIMPSON, W.T., ROSEN, H.N. Equilibrium moisture content of wood at high temperatures. Wood and Fiber, 13(3), 1981, p. 150-158. 
SKAAR, C. Water in wood. New York: Syracuse Univ. Press, 1972.

SKAAR, C. Water in wood. New York: Syracuse Univ. Press, rev. 1988.

WOOD HANDBOOK. Agric. Handb. 72. Washington, DC: U.S. Department of Agriculture; 1955.

WOOD HANDBOOK. Wood as an Engineering Material. Agric. Handb. 72. Washington, DC: U.S. Department of Agriculture; rev. 1987. 